\title{
Feto-Maternal Outcome of HIV Positive Pregnant Women on HAART at the Gambia - Case - Control Study
}

\section{Matthew Anyanwu ${ }^{1,2 *}$, Samuel Anya ${ }^{2}$, Richard Offiong ${ }^{3}$ and Bissallah Ekele $^{3}$}

${ }^{1}$ Edward Francis Small Teaching Hospital, Banjul, The Gambia

${ }^{2}$ School of Medical and Allied Health Sciences, University of The Gambia, The Gambia

${ }^{3}$ University of Abuja Teaching Hospital (UATH) FCT, Nigeria

*Corresponding Author: Anyanwu Matthew, Senior Lecturer, University of the Gambia School of Medicine and Allied Sciences department of Obstetrics and Gynaecology, The Gambia.

DOI: $10.31080 /$ ASWH.2020.02.0127
Received: July 02, 2020

Published: July 30, 2020

(C) All rights are reserved by Matthew

Anyanwu., et al.

\begin{abstract}
Human Immunodeficiency virus (HIV) infection in pregnant women remains a huge public health concern globally. There were conflicting reports regarding pregnancy outcome among HIV positive mothers. The aim was to compare pregnancy outcome among HIV-infected and uninfected women at Edward Francis Small Teaching Hospital, Banjul the Gambia.

Pregnant women attending antenatal clinic at the hospital were prospectively recruited and followed up. HIV positive and negative mothers were matched for age and parity. Recruitment occurred irrespective of gestation age. At the time of delivery, obstetric and neonatal characteristics were entered into computer database. Mothers and their children were followed until 6 weeks postpartum. The data was analyzed with Epi-info version 7.1.5. Chi square at significant level of 0.05 and confidence level of $95 \%$ was used to determine significance.

A total of 166 mothers, 55 HIV positive and 111 HIV negative controls were in the study. The age range was between 18 to 45 years and parity was 0 to 8 . The measured adverse outcome includes; Low Birth Weight; $20 \%$ Vs 5.2\% odds ratio (OR) 4.6; $95 \%$ confidence interval (CI) 1.46-14.2; P- 0.01220 and maternal anaemia (40\% Vs 11\% OR 5.4 95\% CI 2.3 - 12.6; P-0.0001). Both showed statistical significant difference. Preterm delivery; 29.2\% and 26.6\%; OR 1.14; 95\% CI 0.52 - 2.46; P-0.9 and stillbirth, low Apgar score at 5 minutes and NICU admission of 5.45\%vs $0-0.9 \%$ showed no statistical difference; (OR = 6.3; CI; $0.6-6.25$; P -0.21). HIV infection was associated with low birth weight and maternal anaemia.
\end{abstract}

Keywords: HIV; HAART; Pregnancy

\section{Abbreviations}

ANC: Antenatal Clinic; ARV: Anti-retroviral Drug; AZT: Zudovidine; CD4: T4 Lymphocytes; EFSTH: Edward Francis Small Teaching; HAART: Highly Active Antiretroviral Therapy; HIV: Human Immunodeficiency Virus; NICU: Neonatal Intensive Care Unit; PMTCT: Prevention of Mother to Child Transmission of HIV Infection; STD: Sexually Transmitted Disease.

\section{Introduction}

The biological interaction between HIV and pregnancy is not well understood. It has been argued that pregnancy may accelerate HIV progression as pregnancy is associated with suppressed immune function independent of HIV status [1,2]. However, the epidemiological evidence supporting this hypothesis is weak. A 
systematic review investigating the effects of pregnancy on HIV progression and survival found no evidence that pregnancy increased progression to an HIV-related illness or a fall in CD4 count to fewer than 200 cells per cubic milliliter [3].

Some studies have shown significant association of HIV infection and low birth weight [4-7]. However, in the same studies the use of HAART may or may not have played a causative role.

Complications of early pregnancy have also been associated with HIV infection in several studies $[4,5,7,8]$. HIV-1 and HIV-2 infection in Africa have both been linked to a higher rate of spontaneous abortion ${ }^{1}$. However, the impact of HAART was not determined, which is also beyond the scope of this study as to determine the impact of ART on the pregnancy outcome. The dynamic nature of HIV treatment and the evolution of antiretroviral drugs suggest that a cohort study may be required testing for a rare outcome among people living with HIV on different regimen of these ARVs.

However, anaemia has been shown to be more prevalent among HIV positive women [9].

Therefore in view of the conflicting results and variations in study design and methodology we decided to conduct this study with the primary aim to determine the prevalence of stillbirth, preterm delivery, low birth weight, maternal anaemia and low Apgar score in HIV positive pregnant women on HAART during pregnancy and child birth and compare it with the HIV negative pregnant women.

\section{Methodology}

\section{Study location}

Edward Francis Small Teaching Hospital (EFSTH) is the only teaching hospital in the Gambia. It is located in Banjul and being the only Teaching Hospital in the country serves as a referral center for divisional hospitals. The population sub served by this hospital represents approximately $10 \%$ of the country's population of 1.9 Million.

\section{Study design and population}

This was a prospective Case Control study of HIV positive, matched for age and parity with HIV negative women, attending antenatal clinic (ANC) at Edward Francis Small Teaching Hospital and the annexed clinic within greater Banjul.

\section{Sample size}

Prior data from a retrospective survey of pregnancy outcome among HIV Positive mothers in the Gambia between 2005 and 2011 suggests the rate of adverse outcomes of $0.1(10 \%)$ and 0.3 (30\%) among controls (HIV Negative) and experimental subjects (HIV Positive) respectively [10]. Therefore, with probability (power) 0.8 and Type I error probability associated with this test of null hypothesis placed at 0.05 using Power and Sample size (PS) calculation program in 2 (controls) to 1 ( case) ratio the sample size was estimated at 102 HIV negative and 51 HIV positive subjects [11].

Inclusion and exclusion criteria: Pregnant mothers who tested HIV positive during pregnancy or before pregnancy that are currently pregnant and are receiving HAART either for their health or for PMTCT. Mothers who have pregnancy associated medical conditions or preexisting medical conditions such as diabetes, preeclampsia, chronic hypertension and sickle cell disease were excluded.

\section{Procedure}

Awareness about this study was created among hospital staff at the antenatal clinic, labour ward, emergency obstetrics and gynaecology on call duty, outpatient clinics and infectious disease clinic at Edward Francis Small Teaching Hospital. Pregnant women attending antenatal clinic at the hospital from July to October 2015 were prospectively recruited and followed up. Recruitment continued until the sample size was achieved. This included those who had registered for antenatal care and those coming for the first time at any gestation age within the recruitment period. There was no upper limit of gestation age above which recruitment did not occur, however, the exclusion criteria were strictly applied.

Structured interviews were conducted at the first contact to collect information on demographic characteristics, clinical and obstetric history of each participant. The stage of HIV disease was classified in accordance with World Health Organization guidelines [12].

\section{Follow up}

All participants were followed through pregnancy, delivery and up to six weeks postpartum.

Outcome measures: Adverse obstetric outcomes of interest were medically indicated and spontaneous preterm birth (defined 
as delivery before 37 weeks' gestation), maternal anaemia (defined as $\mathrm{HB}<10 \mathrm{~g} / \mathrm{dl}$ or $\mathrm{PCV}<30 \%$ ). Adverse neonatal outcomes of interest includes low birth weight (birth weight $<2500 \mathrm{~g}$ ), Apgar score $<$ 7 at 5 minutes, Number of antepartum and intrapartum stillbirths; and admission into NICU.

\section{Data analysis}

Data tool for each mother was entered into a computer database. Consistent check was ensured to exclude data entering error. A univariate analysis was performed for factors indicated in the primary outcome measures, a test of significance was however, performed with Epi-info version 7.1.5.0. Chi square at significant level of 0.05 and confidence level of $95 \%$ was used to determine significance.

\section{Ethical considerations}

A participant information sheet was used to ensure the provision of adequate information about the study. Informed consent was documented on a consent form and the data collection tools did not capture the participant's name. Those who refused consent were not denied access to quality care. Participants were aware of no monetary incentive attached to their participation. The approval of the study was given by Joint Gambia government and Medical Research Council (MRC) ethics and scientific committee.

\section{Results}

There were 55 HIV positive mothers and 111 HIV negative controls in this study. All were matched for age and parity. The age range was between 18 to 45 years and parity was 0 to 8 .

Table 1 shows demographic characteristics of the study population: Majority of the women studied were in the age range of 25 to 34 years (50-53\%) and predominantly multiparous (62 - 67\%). A few of them were grand multiparous ( $\geq$ Para 4 ) and were appropriately matched to minimize confounding factors. Also many of them had no formal education (62.5\%).

Table 2 shows Pregnancy outcome measure of both HIV positive and negative mothers: Low birth weight (LBW) was defined as birth weight below $2.5 \mathrm{~kg}$. Maternal anemia defined as haemoglobin concentration of less than $10 \mathrm{~g} / \mathrm{dl}$. HIV infection was found to have statistical significant association with low birth weight (0.01220) and maternal anaemia (P-0.0001).

\begin{tabular}{|l|c|c|}
\hline & $\begin{array}{c}\text { HIV positive } \\
\text { n (\%) }\end{array}$ & $\begin{array}{c}\text { HIV negative } \\
\text { n (\%) }\end{array}$ \\
\hline Age (years) & $5(9.1)$ & $11(9.9)$ \\
\hline$<20$ & $10(18.2)$ & $21(18.9)$ \\
\hline $20-24$ & $28(50.9)$ & $59(53.5)$ \\
\hline $25-34$ & $8(14.5)$ & $12(10.8)$ \\
\hline$>35$ & $4(7.3)$ & $8(7.2)$ \\
\hline Unknown & 55 & 111 \\
\hline Total & $14(25.5)$ & $31(27.9)$ \\
\hline Parity & $17(30.9)$ & $38(34.2)$ \\
\hline $0-1$ & $18(32.7)$ & $37(33.3)$ \\
\hline $2-4$ & 2 & 5 \\
\hline$>4$ & $55(100)$ & $111(100)$ \\
\hline Unknown & & \\
\hline Total & $6(10.9)$ & $7(6.3)$ \\
\hline Level of education & $10(18.2)$ & $15(13.5)$ \\
\hline Primary & $20(36.4)$ & $3(2.7)$ \\
\hline Secondary & $55(100)$ & $(26.1)$ \\
\hline Tertiary & & \\
\hline None & & $(51.4)$ \\
\hline Unknown & & \\
\hline Total & & \\
\hline
\end{tabular}

Table 1: Sociodemographic characteristics of the study population.

\begin{tabular}{|l|c|c|c|c|c|}
\hline & $\begin{array}{c}\text { HIV POS } \\
\mathbf{n ( \% )}\end{array}$ & $\begin{array}{c}\text { HIV NEG } \\
\mathbf{n ( \% )}\end{array}$ & P-Value & OR & $\mathbf{9 5 \% ~ C I ~}$ \\
\hline $\begin{array}{l}\text { Low birth } \\
\text { weight }\end{array}$ & $10(20)$ & $5(5.2)$ & 0.01220 & 4.6 & $1.46-14.2$ \\
\hline $\begin{array}{l}\text { Maternal } \\
\text { anaemia }\end{array}$ & $22(40)$ & $10(11)$ & 0.0001 & 5.4 & $2.3-12.6$ \\
\hline $\begin{array}{l}\text { Preterm } \\
\text { delivery }\end{array}$ & $14(29.2)$ & $25(26.6)$ & 0.9 & 1.14 & $0.52-2.46$ \\
\hline Stillbirth & $3(5.45)$ & $1(0.9)$ & 0.21 & 6.3 & $0.6-6.25$ \\
\hline $\begin{array}{l}\text { Low Ap- } \\
\text { gar score }\end{array}$ & $3(5.45)$ & 0 & 0.21 & 6.3 & $0.6-6.25$ \\
\hline $\begin{array}{l}\text { NICU ad- } \\
\text { mission }\end{array}$ & $3(5.45)$ & 0 & 0.21 & 6.3 & $0.6-6.25$ \\
\hline
\end{tabular}

Table 2: Pregnancy outcome measure of both HIV positive and negative mothers. 
Preterm delivery defined as delivery before 37 completed weeks of gestation. There was no statistical significance difference between the two groups (P-0.9). Similarly, there was no difference in the incidence of stillbirth, low Apgar score at 5 minutes and NICU admission (P-0.21).

Figure 1 A linear regression analysis of our data using level of education as a proxy of socioeconomic factor to assess rate of low birth weight in both HIV positive and negative mothers. There was no significant association between HIV positive and Negative mothers levels of education in relation to low birth weight.

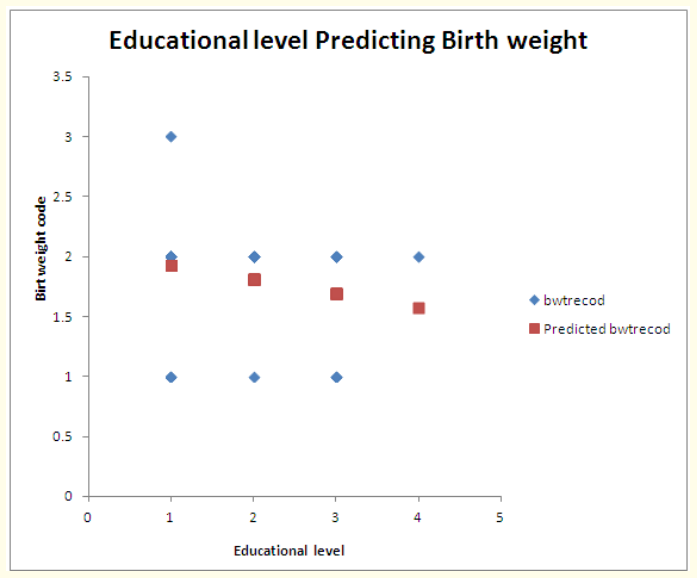

Figure 1: Regression analysis for confounders such as educational level (a proxy of socioeconomic factor) did not predispose to low birth weight in the HIV positive and negative cohort.

Figure 2 HIV positive status was a strong factor to low birth weight.

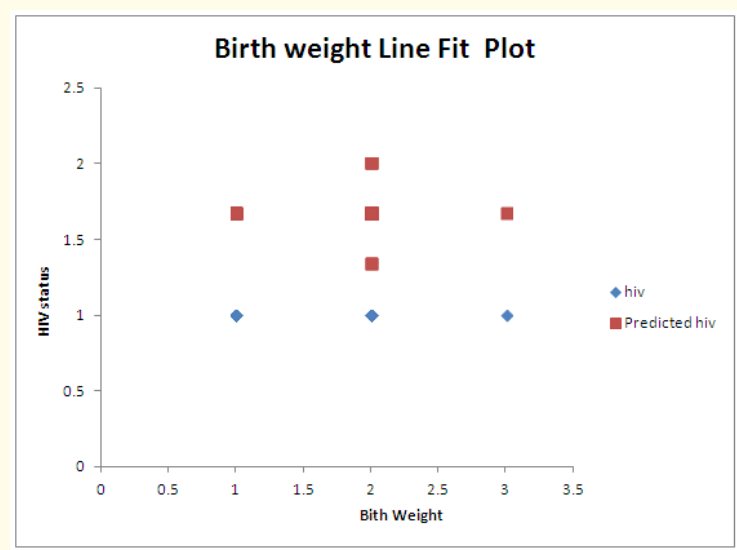

Figure 2: HIV predicting probability of low birth weight.

\section{Discussion}

Majority of the women studied were in the age range of 26 to 35 years (53\%) and predominantly multiparous (68.6\%). The age range is similar to other studies conducted elsewhere $[7,13,14]$ which may explain strong sociocultural similarities and the importance of child birth despite increasing maternal age. However, high parity among HIV positive mothers is a remarkable finding in this study. This is contributing to the body of evidence that HIV infection do not adversely affect fertility. A systematic review of the literature and meta-analysis by French., et al. [15] on the effect of pregnancy on survival in women infected with HIV concluded that fertility rate is not affected by HIV infection.

HIV infection was found to be associated with low birth weight. A Rwanda study [5] which was similar to our study concluded that birth weight was significantly lower in singleton babies of HIV infected asymptomatic women than in babies born to uninfected women.

Other studies in predominantly asymptomatic cohorts have shown no significant difference in birth weights such as a study conducted in North-Eastern Nigeria by Ahmadu., et al. [8] it was a hospital-based randomized descriptive comparative study on birth weight outcome of babies whose mothers were infected with H I V and on antiretroviral therapy. The result of the study showed no significant difference between the two groups ( $p=0.419)$. Although, the sample size was small; 90; 45 HIV positive and 45 matched HIV negative. The power calculation methodology adopted in our study ensured mitigation of Type 1 error as the control group was 2-fold the positive cases.

The study conducted at the HIV treatment centre, Nigerian Institute of Medical Research, Lagos by Ezechi., et al. [7] investigated pregnancy and neonatal outcome of HIV positive women over a period of 84 months. The study concluded that, Low birth weight, preterm delivery, perinatal death and spontaneous abortion were significantly associated with HIV related severe immunosuppression and opportunistic infection. What was remarkable in the Ezechi., et al. study was that the adverse pregnancy outcome was linked to advanced disease which is similar with a study conducted in Nairobi, which concluded that HIV-infected women were 3 times more likely to deliver a low-birth-weight baby; especially in the presence of HIV-related symptoms [4]. In view of the available literature, symptomatic HIV infection is more likely than not to be associated with low birth weight, however, in asymptomatic HIV infection is as yet inconclusive.

HIV positive factor was strongly associated with low birth weight. 
A linear regression analysis of our data using level of education as a proxy of socioeconomic factor did not have any significant impact on the rate of low birth weight in both HIV positive and negative mothers (Figure 1). However, HIV positive condition was a strong factor to low birth weight (Figure 2). Therefore, our study suggests HIV infection may be associated with low birth weight in asymptomatic HIV positive pregnant women.

A large population study in Nairobi showed an independent association between HIV infection and intrauterine and intrapartum death after controlling for the presence of other sexually transmitted disease (STDs) [4]. Previous studies have similar results especially in advanced disease, where reduced fecundity, miscarriages and stillbirths were reported [5,16-18]. Our study did not suggest any difference between the two groups in these adverse pregnancy outcomes.

The result did not show any difference in preterm birth between HIV positive and negative cohort. However, anaemia has shown to be more prevalent among HIV positive women. Some other study conducted elsewhere have similar result that anemia is associated with HIV infection [9]. This may be due to some ARV drugs such as AZT which majority of the patients were on it (81.8\%). The disease may also predispose to anaemia.

There were conflicting results regarding the effect of HIV infection on Apgar score and admission into NICU. In the study by Tuomala., et al. [19], Apgar score and increased neonatal admissions were not associated with HIV infection which was similar to the results of our study.

\section{Study Limitation}

Duration of data collection, sample size and limited cofounders' such as duration on ARV may have implication on the results of the study. The viral load machine was out of service during the study period.

\section{Conclusion}

HIV infection is associated with low birth weight and maternal anaemia. Majority of the patients were multigravida, little or no formal education and high parity. These are independent risk factors of maternal anaemia, low birth weight and poor obstetrics outcome. As these confounding factors were minimized in the study by appropriate matching of age and parity, therefore HIV infection could be an independent risk factor of these feto-maternal morbidities.

\section{Recommendation}

The findings of this study suggest low birth weight and maternal anaemia were associated with HIV infection. Therefore further research is required to determine the impact of multivitamin supplement during pregnancy for HIV positive cohort on anaemia and low birth weight in our setting.

\section{Authors' Contributions}

AM conceived the idea of the study and participated in its design. AM developed the data collecting tool and piloted it. AM supervised data entry into a dedicated database. AS analysed data. AM wrote the first draft of the manuscript. OR and EB reviewed all drafts of the manuscript and made corrections. All authors read and approved the final manuscript.

\section{Availability of Data and Materials}

The datasets generated and/or analyzed during this study are available from the corresponding author on reasonable request. No personal or individual data in this manuscript or database.

\section{Bibliography}

1. Lindgren S., et al. "Pattern of HIV viraemia and CD4 levels in relation to pregnancy in HIV-1 infected women". Scandinavian Journal of Infectious Diseases 28 (1996): 425-433.

2. Rich KC., et al. "CD4+ lymphocytes in perinatal human immunodeficiency virus (HIV) infection: evidence for pregnancyinduced immune depression in uninfected and HIV-infected women". Journal of Infectious Diseases 172 (1995): 1221-1227.

3. French R and Brocklehurst P. "The effect of pregnancy on survival in women infected with HIV a systematic review of the literature and meta-analysis". BJOG: An International Journal of Obstetrics and Gynaecology 105 (1998): 827-835.

4. Temmerman M., et al. "Department of Medical Microbiology, University of Nairobi, Kenya”. Obstetrics and Gynecology 83.4 (1994): 495-501.

5. Leroy V., et al. "Effect of HIV-1 infection on pregnancy outcome in women in Kigali, Rwanda, 1992-1994. Pregnancy and HIV Study Group". AIDS 12 (1998): 643-650. 
6. Lorenzi P. "Antiretroviral therapies in pregnancy: maternal, foetal and neonatal effects. Swiss HIV Cohort Study, the Swiss Collaborative and Pregnancy Study, and the Swiss Neonatal HIV Study". AIDS 12 (1998): 241-247.

7. Ezechi OC., et al. "Pregnancy, Obstetric and Neonatal Outcomes in HIV Positive Nigerian Women". African Journal of Reproductive Health 17.3 (2013): 160-168.

8. Ahmadu BU., et al. "Birth weight outcome of babies whose mothers are infected with Human Immunodeficiency Virus and on antiretroviral therapy at University of Maiduguri Teaching Hospital, Nigeria". Greener Journal of Medical Sciences 3.2 (2013): 053-056.

9. Adesina O., et al. "Risk of anaemia in HIV positive pregnant women in Ibadan, south west Nigeria". African Journal of Medicine and Medical Sciences 40.1 (2011): 67-73.

10. Anyanwu M., et al. "HIV infection: The course and outcome of pregnancy in The Gambia between 2005 and 2011 - a descriptive cross sectional survey". UNAIDS The Gambia (2013).

11. Dupont WD and Plummer WD. "Power and Sample Size Calculations: A Review and Computer Program". Controlled Clinical Trials 11 (1990): 116-128.

12. Interim WHO clinical staging of HIV/AIDS and HIV/AIDS case definitions for surveillance African region (2005).

13. Olagbuji BN., et al. "Obstetric and perinatal outcome in HIV positive women receiving HAART in urban Nigeria". Archives of Gynecology and Obstetrics (2009): 281.

14. Onah HE., et al. "Pregnancy outcome in HIV-positive women in Enugu, Nigeria”. Journal of Obstetrics and Gynaecology 27 (2007): 271-274.

15. French R and Brocklehurst P. "The effect of pregnancy on survival in women infected with HIV a systematic review of the literature and meta-analysis". BJOG: An International Journal of Obstetrics and Gynaecology 105 (1998): 827-835.

16. Fawole AO., et al. "HIV in pregnancy: Experience at Abeokuta Nigeria". Tropical Journal of Obstetrics and Gynaecology 1 (2002): 7-10.

17. Cotter AM., et al. "Is antiretroviral therapy during pregnancy associated with an increased risk of preterm delivery, low birth weight, or stillbirth". Journal of Infection Disease 193.9 (2006): 1195-1201.
18. Townsend CL., et al. "Antiretroviral therapy and premature delivery in diagnosed HIV-infected women in the United Kingdom and Ireland". AIDS 21 (2007): 1019-1026.

19. Tuomala R., et al. "Antiretroviral Therapy during Pregnancy and the Risk of an Adverse Outcome". The New England Journal of Medicine 346 (2002): 1863-1870.

\section{Assets from publication with us}

- Prompt Acknowledgement after receiving the article

- Thorough Double blinded peer review

- Rapid Publication

- Issue of Publication Certificate

- High visibility of your Published work

Website: $\underline{w w w . a c t a s c i e n t i f i c . c o m / ~}$

Submit Article: www.actascientific.com/submission.php Email us: editor@actascientific.com

Contact us: +919182824667 